\title{
Infection prevention training of trained birth attendants and nurse supervisors in primary health centres in rural Cameroon
}

\author{
Nwenfu Peter Kakute ${ }^{1}$, Dorothy J. Meyer ${ }^{2}$, Nkwan Jacob Gobte ${ }^{3}$ \\ ${ }^{1}$ LAP Director \\ ${ }^{2}$ CAPT. USPHS, Ret. Consultant, \\ ${ }^{3}$ Department of Nursing, Faculty of Health Science, University of Buea, Cameroon
}

doi: 10.3396/ijic.V7i4.035.11

\begin{abstract}
The Cameroon Baptist Convention Health Board (CBCHB) Life Abundant Primary health care program (LAP) received the 2010 IFIC-Covidien Grant. The primary project objectives were (1) to provide infection prevention training to Trained Birth Attendants (TBAs) who deliver women in rural Primary Health Centres (PHC) and to Nurse Supervisors who supervise and monitor the medical and maternity care provided in the PHCs, and (2) to implement pilot infection prevention programs in 5 PHCs. The training pre-test showed that $15 \%$ of the TBAs had adequate knowledge of infection prevention. Upon completion of the training, $82.5 \%$ of TBAs exhibited adequate knowledge of infection prevention on the post-test. The Nurse Supervisor's knowledge also increased from $54.5 \%$ having adequate knowledge on the pre-test to $100 \%$ upon the post-test. Infection Prevention Pilot Programs were implemented in 5 PHCs with each PHC maintaining appropriate infection prevention practices and equipment/supplies after 6 months.
\end{abstract}

\section{Introduction}

Cameroon is a country in sub-Saharan Africa with a population estimated at more than 19.5 million (2009). ${ }^{1}$ It consists of many tribal groups, each with its own unique language and culture. The climate varies with the terrain, from tropical forests along the coasts to semiarid and hot in the north. It is estimated that
$56 \%$ of deliveries occur in rural areas of Cameroon with $44.2 \%$ of rural women delivered by skilled birth attendants. ${ }^{2}$ In 2008, the Cameroon Maternal Mortality ratio was estimated to be 705 per 100,000 live births with an Infant Mortality Rate of 74 per 1000 live births. ${ }^{3}$

\section{Corresponding author}

Dorothy J. Meyer

7409 Bullion Hill Road, Flagstaff, Arizona, USA

Email: DotJMeyer@aol.com 
The Cameroon Baptist Convention Health Board $(\mathrm{CBCHB})$ is a private, faith-based health care system consisting of 5 hospitals, 26 Integrated health centres $(\mathrm{IHC})$, and 46 primary health centres (PHC). Since 1980, the CBCHB has provided rural health care services through the Life Abundant Primary health care program (LAP). ${ }^{4} \quad$ LAP has now expanded health education and basic outpatient care services to 46 community-sponsored and maintained primary health centres (PHCs) located in rural and isolated villages. Maternity care was added to the PHC services in 1990, and LAP maternity care has grown from 646 women delivered in 18 PHC service areas (1991) to 1082 women delivered in 40 PHCs (2010).

\section{Background}

The Life Abundant Primary health care program: The mission of LAP is to provide health care to those rural areas which would not otherwise have access to even basic health education and medical care services. The majority of the villages with PHCs are very remote. If there is a road to the village, it is often impassable. It is not uncommon that a PHC village can only be reached by a 1-3 day "trek". The population of these rural villages range from 500 to 3000 .

The LAP PHC system of medical and obstetrical care consists of 3 interdependent components:

- The PHC is built, maintained and managed by a local Village Health Committee which has the final approval of all services and activities. LAP administrative staff provides support to and supervision of the PHC s financial and daily work affairs to the Village Health Committees, as well as ongoing education.

- The PHC is staffed by health promoter(s) and Trained Birth Attendant(s) (TBAs). Using standing orders, the health promoter provides limited outpatient medical care treating common illnesses. All maternity care is provided at the $\mathrm{PHC}$ by the TBAs, including antenatal, labour and delivery, and postpartum care. These village health care workers are selected by the community and reside within the community. To be accepted for TBA training, the individual must be literate, wellrespected in the community, of positive moral attitude, and have given birth or fathered babies. Though most of the TBAs are female, gender is not a selection criterion. The program has become so acceptable that most village women now give birth at their local PHC.

- The LAP provides both initial and continued training plus ongoing supervision and technical support to the Village Health Committees and PHC staff through Nurse Supervisors. These nurses visit each $\mathrm{PHC}$ on a regular basis to monitor and supervise the care provided. On their periodic visits, the Nurse Supervisors also administer immunizations and evaluate the more complicated patients. Each year, all health promoters and TBAs minimally receive 3-5 days of education at the LAP central Office.

The CBCHB Infection Control Program: In 2002, the $\mathrm{CBCHB}$ Infection Control Program was initiated in hospitals and health centre's in response to the recognition of persistent in-hospital newborn "septic" skin infections and documented consultant concerns regarding observed poor infection control practices. The Infection Control Program was developed from and based upon the Engender Health Infection Program guidelines and recommendations. ${ }^{4}$ All new physicians, nurses, and ancillary staff are invited to an eight hour infection prevention training as part of their orientation. Each attendee is asked to complete a pre and post-test to assess their initial knowledge as well as comprehension of infection control practices upon course completion. This test consists of 20 questions with a correct score of $16(80 \%)$ considered exhibiting adequate knowledge. Further (2-3 day) infection prevention and control in-services are provided to staff throughout each year.

The staff of the Infection Control program supervises and monitors infection prevention and control practices in all hospitals and clinics.

\section{Project description}

The Covidien Grant project objective was "to integrate Infection Prevention Services and Training into the LAP Primary Health Care Program of the Cameroon Baptist Convention Health Board." Basic infection prevention practices are taught in the initial TBA training and are continually assessed by the Nurse Supervisors at their on-site visits. Yet, the actual observed cleanliness of the PHCs and infection prevention practices of 
the PHC staffs are variable and dependent upon the local Village Health Committee's understanding and support, as well as the knowledge, attitude, and practice of the individual PHC staff members. Our goal of an "organizational" culture and expectation of cleanliness and infection prevention in PHCs has remained elusive. Meeting this goal is made further challenging, because piped water is only available in 3 of the 40 PHCs (7.5\%) with maternity services.

This project began in January 2010 and was divided into 2 phases (January to June/ July to December). The project goals consisted of:

- providing infection prevention training to TBAs and Nurse Supervisors, and

- implementing a pilot infection prevention program at 5 PHC's.

Phase 1 - Infection Prevention Training: It was selected to use the same CBCHB Infection Control training provided to hospital and clinic staff. The training was conducted by the LAP Program Director and $\mathrm{CBCHB}$ Infection Control Program. Two (4 day) training sessions were held for 2 groups of TBA's. One (two day) training session was held for Nurse Supervisors. The CBCHB Infection Control Program's pre and post-test was used to assess each participant's infection prevention knowledge before and after the training. At the end of the training, infection prevention action plans were developed for their individual PHCs by all TBA participants. The Nurse Supervisors also developed infection prevention action plans for their service area.

\section{Phase 1- Infection Prevention PHC Pilot Program:} LAP and the Infection Control Program staff developed guidelines for implementing defined infection prevention activities at a PHC. This included (1) identifying a listing of equipment and supplies essential to infection prevention programs as well as (2) educational activities appropriate to Village Health Committees and community understanding of infection prevention. Five PHCs were selected to pilot the infection prevention program activities. An infection prevention program was explained to each of the selected PHC Village Health Committees and their support and permission to pilot the program was requested. Once permission was obtained, a one day
Table I. Recommended equipment and supplies which should be available at PHCs where births take place.

1. Five sterile surgical pairs of gloves.

2. One box of examination gloves (\# 100).

3. One litre of prepared alcohol for hand rub after an examination of a client. (Preparation consists of $2 \mathrm{ml}$ of glycerine oil to $100 \mathrm{ml}$ of $60-90 \%$ ethyl or isopropyl alcohol.)

4. One $3 \mathrm{ml}$ container to dispense alcohol hand rub liquid.

5. One 14 litre transparent rubber bucket with lids and with head tape locally made for running water to wash hands in the delivery room.

6. One wooden stand for the bucket of water.

7. Five cubes of savon soap.

8. Five cubes of antiseptic soap.

9. One size 3 "cement" pot for sterilization of equipment (by boiling). (A "cement" pot is made of aluminum sheet zinc).

10. One small size kerosene stove for fire (fuel).

11. One 100 cc container of Povidine 10\% solution.

12. Each site will purchase bleach locally to make a $0.5 \%$ solution.

on-site infection prevention training was provided by the LAP Director and Infection Control Program staff. The PHC staff, Village Health Committee, and community members were invited to attend. At the completion of this training, equipment and supplies (table I) were distributed to the PHC staff.

Phase 2 - Infection Prevention Training: The LAP Director and Infection Control Program staff also served as trainers in Phase 2. Two (2 day) training sessions were held for TBA's participants. A one day training session was held for Nurse Supervisors.

All Phase 1 participants were invited to return for Phase 2 training. Those, who had been unable to attend Phase 1 training, were re-invited to Phase 2 training. Again, an assessment of infection prevention knowledge was performed by administering the CBCHB Infection Control Program pre and post-test. Positive infection prevention practices were again presented. The infection prevention action plans 
developed in the Phase 1 training by participants were reviewed and discussed with successes and challenges identified at each participant's PHC or service area.

Phase 2 - Infection Prevention Pilot Program: In December 2010, the 5 pilot PHCs were each visited by the LAP Director and Infection Control Program staff. Each pilot program was evaluated as to their implementation and maintaining of infection prevention practices, equipment and supplies.

\section{IFIC-Covidien grant project results}

TBA Training: Four training sessions for TBAs were held: two in Phase 1 and two in the Phase 2. Training participants represented 30 of the 40 PHCs with TBAs (75.0\%). Ten PHCs were unable to send participants for various reasons, including inability to obtain transportation or health concerns. There were 53 TBAs eligible to attend the training with $39(73.6 \%)$ participating in at least one training session. Of these 39 TBAs, 21 participated in a training session in both Phase 1 and 2. Seven attended training only in Phase 1 and eleven only in Phase 2.

The knowledge of the TBA participants was shown to increase:

- The average score of the first pre-test administered to TBA participants was 13.5 , with a range of 7 to 18.

- The average score of the final post-test administered to TBA participants was 17.3 , with a range of 11 to 20.

- $\operatorname{Six}(15.4 \%)$ TBA participants demonstrated adequate infection prevention knowledge on their initial pre-test (answered $80 \%$ of questions correctly).

- Thirty-three (84.6\%)TBA participants demonstrated adequate infection prevention knowledge on their final post-test (answered $80 \%$ of questions correctly).

- Five (12.8\%) TBA participants achieved a 100\% correct score on the final post-test, as compared to no TBA scoring $100 \%$ on the initial pre-test.

In the final training, the TBA participants worked in groups to identify where their local infection prevention efforts should be focused. The following were identified:
1. Correct hand washing technique

2. When to wash hands

3. The need to institute and maintain a PHC cleaning schedule

4. The 3 cleans of delivery (clean hands, clean cord cutting, clean delivery area)

5. How to clean and maintain clean instruments

Nurse Supervisor Training: One training session for Nurse Supervisors was held in Phase 1 and one in Phase 2. There are ten (10) Nurse Supervisors. Eight were able to attend the Phase 1 training with 9 attending the Phase 2 training. All Nurse Supervisors attended at least one training session. The Nurse Supervisors also showed an increased knowledge of infection prevention practices. In their initial pre-test, $54.5 \%$ exhibited adequate infection prevention knowledge (answered $80 \%$ of questions correctly). In their final post-test, all Nurse Supervisors demonstrated adequate knowledge with $46.1 \%$ scoring $100 \%$.

In the final training, all Nurse Supervisors identified three general areas where they would focus further efforts:

1. hand hygiene,

2. waste management, and

3. housekeeping.

In addition, 5 nurse supervisors identified plans to address equipment and supply issues, 3 water management, and 2 the education of the community and Village Health Committee.

Pilot Projects: In Phase 1, the Infection Prevention education was provided to the Village Health Committee, PHC staff, and community members by the Infection Control Program staff and LAP Director. At the end of the session, the equipment and supplies were given to the PHC staff.

In December 2010 (Phase 2), the 5 demonstration sites were visited by the LAP Director and Infection Control Program staff with the following observed:

- Each PHC site maintained and used their equipment and supplies consistent with infection prevention practices. This included the posting of a cleaning schedule which was respected with documented daily cleaning, as well as the orderliness in the 
storage of supplies and equipment.

- An appropriate method of refuse disposal was developed and maintained at each PHC.

- The " 3 " cleans ${ }^{6}$ (which is taught in all TBA trainings) was observed in one PHC during a delivery by a TBA.

\section{Discussion}

We believe that the test results demonstrate that the training provided TBA participants an adequate knowledge of infection control practices as well as promoting positive attitudes concerning implementation and use of infection prevention practices. There is no doubt that all the TBA participants are now attempting to put improved infection prevention practices into place. Yet, the challenge remains to sustain infection prevention practices and avoid the return to previously observed inadequate practices and habits.

Unique to LAP is the ongoing support, monitoring and encouragement of Nurse Supervisors. Their work is pivotal to sustaining an acceptable quality of service at each PHC. The Nurse Supervisors also received infection prevention training as well as training in the monitoring of infection prevention activities. Their ongoing efforts are essential to sustaining adequate infection prevention practices at each of the PHCs. We believe that this training has renewed the Nurse Supervisor's support of the importance of infection control with both the PHC staff and Village Health Committees.

The Nurse Supervisors have a check sheet which they complete and submit to LAP administration after each $\mathrm{PHC}$ visit. Some infection prevention practices are addressed in this evaluation. LAP administration will review this form. Either a new check sheet will be developed, or the present evaluation system will be modified to focus upon specific infection prevention practices. All Nurse Supervisors attend bi-annual management meetings. Infection control, successes and challenges, will be discussed as a regular agenda item at each meeting. In time, we anticipate that an "organizational culture" of infection prevention/ cleanliness will be achieved.
Finally, we believe that the action plans developed at the training sessions demonstrate an enhanced knowledge and understanding of infection prevention on both an individual and community basis.

It was not only important to develop initial action plans, but the follow up of success and challenges with all participants in the final training sessions was extremely helpful in identifying where further program efforts should be focused to support infection prevention activities.

In the Phase 2 training, the TBAs were asked to review and revise their individual $\mathrm{PHC}$ action plans.

- The TBAs of 18 PHCs identified the persistent lack of recommended equipment and supplies.

The PHC Village Health Committee is responsible for providing equipment and supplies to the PHC. The Nurse Supervisors will bring this issue to the attention of the Village Health Committees and support the staff's requests to obtain the recommended equipment and supplies. In time, we hope that every PHC will have the equipment and supplies furnished by the Village Health Committees.

- Addressing the continued challenges of adequate waste management, including general waste and placenta disposal, was identified within 18 TBA PHC final action plans.

The PHC Village Health Committee is responsible for instituting acceptable waste management activities. The Nurse Supervisors will bring this issue to the attention of the Village Health Committees and support the staff's requests to institute appropriate waste management activities. In time, we hope that each PHC will have acceptable waste management.

- Instituting and/or maintaining adequate hand washing and housekeeping was identified within 19 TBA PHC final action plans. Eight specifically identified expanding infection control in the community with the goal of providing community education on hand washing. 
Hand hygiene is the cornerstone of infection prevention. All PHC and LAP staff will be encouraged to follow the LAP hand washing guidelines and to serve as an example to their communities with an ongoing educational emphasis on hand washing.

\section{Conclusion}

Based on the above, we believe that the project goal has been met. We plan to continue to place emphasis upon infection prevention practices with the hope of developing the expectation of ongoing and consistent infection prevention practice at each $\mathrm{PHC}$.

\section{Added comment}

In March 2010, the Trained Birth Attendant title was formally changed to "Community Mother and Child Health Aide" (CoMCHA). Since the inception of the TBA Program in 1989, the role and responsibilities of the TBAs have evolved and expanded to include many additional aspects of maternal, women's, and child health care. These expanded and increased responsibilities were not reflected within and recognized by the TBA title. This name change also helps to address the persistent confusion by some of the title "Trained" Birth Attendant with that of a "Traditional" Birth Attendant. A "Traditional Birth Attendant" does not have the initial and ongoing training, as well as monitoring and supervision of the LAP Trained Birth Attendants (now CoMCHAs).

\section{References}

1. US Department of State, Bureau of African Affairs, Background Notes: Republic of Cameroon. April 22, 2011. http://www. state.gov/r/pa/ei/bgn/26431.htm

2. World Health Organization, Cameroon Country Profile, Making Pregnancy Safer, 2007. World Health Organization, Geneva Switzerland. http://www.who.int/making_pregnancy_ safer/countries/en/

3. Hogan MC, Foreman KJ, Naghavi M, et al. Maternal mortality for 181 countries, 1980-2008: a systematic analysis of progress towards Millennium Development Goal 5. The Lancet 2010; 375: 1609-1623. http://dx.doi.org/10.1016/ S0140-6736(10)60518-1

4. Eliason RE. Towards sustainability in village health care in rural Cameroon. Health Promotion International 1999; 14(4): 301-306. http://dx.doi.org/10.1093/heapro/14.4.301

5. EngenderHealth, "Infection Prevention-A reference booklet for health care providers", 2001. http://www.engenderhealth. org/files/pubs/qi/ip/ip-ref-eng.pdf

6. UNFPA, "Inter-agency Field Manual on Reproductive Health in Refugee Situations", 1995 with revision 1999. http://www. unfpa.org/emergencies/manual/index.htm 